\title{
Primary Omental Torsion Mimicking Acute Appendicitis: An Unusual Cause of Acute Abdominal Pain in a Young Male
}

\author{
Mehmet Tolga Kafadar' (D), Ismail Cetinkaya' (D), Osman Bardakci² (D) \\ 'Clinic of General Surgery, Health Sciences University Mehmet Akif Inan Training and Research Hospital, Sanliurfa, Turkey \\ ${ }^{2}$ Department of General Surgery, Harran University School of Medicine, Sanliurfa, Turkey
}

Cite this article as: Kafadar MT, Cetinkaya I, Bardakci O. Primary Omental Torsion Mimicking Acute Appendicitis: An Unusual Cause of Acute Abdominal Pain in a Young Male. Eurasian J Emerg Med. 2018; 17 (3): 136-8.

\begin{abstract}
Omental torsion is a very rare cause of acute abdomen. It commonly indicates itself with the symptoms and signs of acute appendicitis because of its localization on the right of the abdomen. The diagnosis is usually finalized by performing laparotomy or laparoscopy. Omental torsion may be primary or secondary, depending on omental cyst, tumor, intra-abdominal adhesions, or hernias. In this article, we present a 27-year-old male patient who underwent emergency surgery with a preliminary diagnosis of acute appendicitis and postoperatively diagnosed to have omental torsion. Considering omental torsion and different pathologies in patients with suspected clinical acute appendicitis, we are of the opinion that it would be useful to consider alternatives such as different surgical incisions and diagnostic laparoscopy.
\end{abstract}

Keywords: Acute abdomen, appendicitis, omentum, torsion

\section{Introduction}

Omental torsion is a very rare cause of acute abdomen. Necrosis can emerge in the omental tissue with torsion secondary to ischemia. Depending on the localization of the necrotic omentum, it can mimic acute appendicitis, acute cholecystitis, peptic ulcer perforation, diverticulitis, or renal colic clinic. Symptoms are non-specific, and hence it is hard to preoperatively diagnose it (1). In this article, we investigated a young male patient who was diagnosed with intraoperative omental torsion in our clinic, in the light of literature data.

\section{Case Presentation}

A 27-year-old male patient was admitted to the emergency department with complaints of abdominal pain and nausea persisting for 3 days. The patient had no history of any surgical operation or disease. The patient had a pulse of 84 beats/min, blood pressure of $100 / 60 \mathrm{mmHg}$, and a temperature of $37.3^{\circ} \mathrm{C}$. The right inferior abdominal quadrant was associated with tenderness, defense, and rebound during the physical examination. Laboratory tests results showed $12.500 / \mathrm{mm}^{3}$ white blood cell and $15 \mathrm{mg} / \mathrm{dL}$ C-reactive protein, and the other biochemical parameters were found to be normal. Minimal free fluid was observed in the periphery of the intestinal loop in the abdominal right inferior quadrant on the ultrasonography (US) conducted because of the preliminary diagnosis of acute appendicitis. Abdominal tenderness induced by compression of the probe was observed, and a suspicious condition was identified for acute appendicitis. On abdominal computed tomography (CT), the mesenteric adipose planes were contaminated and inflamed; however, no clear tubular formation was detected in the ileocecal region (Figures $1 \mathrm{a}$ and $\mathrm{b}$ ). The patient was operated with the preliminary diagnosis of acute appendicitis based on the current findings. The abdominal cavity was accessed through a Mc Burney incision, and the appendix of the patient was found to be normal. Intra-abdominal serohemorrhagic fluid of approximately 200 cc was observed, and an abdominal mid-line incision that turns around the umbilicus was made to reveal the etiology clearly. During exploration, the omentum, with approximate dimensions

ORCID IDs of all authors: M.T.K. 0000-0002-9178-7843; I.C. 0000-0001-7081-2344; O.B. 0000-0003-0067-3451. 


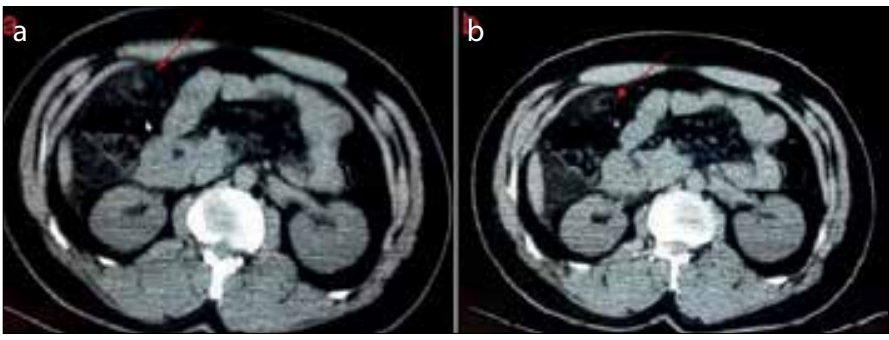

Figure 1. a, b. Computed tomography scan images of the patient
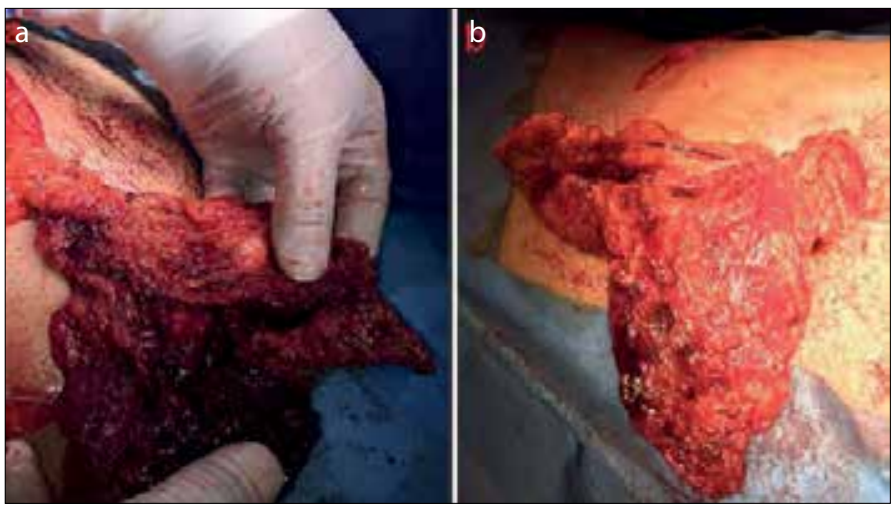

Figure 2. a, b. Intraoperative view of the twisted omentum showing ischemia and necrosis

of $12 \times 8 \times 5 \mathrm{~cm}$ and twisted around itself with a narrow pedicle and distorted circulation and not adhered on any place from its distal, was seen on the right and then excised (Figures $2 a$ and b). No other pathology was seen in the intra-abdominal organs and the abdomen was irrigated, following which appendectomy was performed, and the operation was terminated. The patient was discharged with full recovery after 3 days of hospitalization because no problems or complications were observed. Necrosis and hemorrhagic foci of the omental tissue were reported in the postoperative histopathological examination. Informed consent was obtained from the patient who participated in this case.

\section{Discussion}

Omental torsion is a very rare clinical condition having commonly peritonitis signs, in which edema, vascular stasis, ischemia, and necrosis are seen distal to the torsion because of the rotation of the omentum magus around the long axis and deterioration of arterial feeding and venous drainage secondary to this. The omental torsion, which has a small share among the emergency surgical procedures, was first described by Eitel in 1899 (2).

Omental torsion is divided into primary (idiopathic) and secondary. The more common secondary omental torsion is usually caused by adhesions, omental cysts, tumors, and hernias. Anatomic variations such as accessory omentum, bifid omentum, omental venous malformations, and obesity are included in the primary omental torsion (POT) as predisposing factors (3). Anatomical variations in the vascular structures, venous malformations that may cause stasis or thrombosis, and venous congestion emerging after an excessive and heavy meal are among the suggested reasons for primary omental infarction (4).
Omental torsion often occurs in the $4^{\text {th }}$ and $5^{\text {th }}$ decades and usually in males. The occurrence of omental torsion on the right flank more commonly leads to difficulties in differential diagnosis from diseases such as acute appendicitis, acute cholecystitis, and cecal diverticulitis. The reason of occurrence of omental torsion on the right flank more commonly is that the omentum is longer and larger on the right side than on the left side. Omental torsion develops in two ways. The first is in the form of torsion of a piece of omentum with a large volume around a fixed point and the second is in the form of rotation of the omentum around a narrow neck (5). The type of omental torsion in our patient is of the second form.

In omental torsion, patients often get admitted because of complaints of abdominal pain. According to anamnesis, sudden onset of pain accompanied by trauma, excessive exercise, sudden movements, coughing and strain, and gradual worsening of these symptoms may suggest omental torsion. In addition, pain not accompanied by nausea, vomiting, or lack of appetite in many patients may be important in terms of differential diagnosis. In omentum torsion, pain is felt in the region where torsion occurs in association with the localization of the segment with torsion and often on the right flank. Many literature reports have suggested that the diagnosis is usually made intraoperatively because the symptoms are non-specific (6). Ultrasound and CT can be rarely useful. An oval-shaped, non-compressed, hyperechoic solid mass and a hypoechoic ring surrounding it may be detected on ultrasound, especially in the region of tenderness, just below the anterior abdominal wall. Again, on ultrasound, minimal fluid might be detected although the appendix appears normal. No pathology other than minimal fluid was detected on the patient's ultrasound. In the CT examination, it appears as a heterogeneous fatty mass in front of the intestines. Concentric circular lines may be seen in the middle of the mass because of damages in the vascular structures. These lines are named as whirl sign, and they are pathognomonic for the torsion of the omentum (7).

The POT complications are omental necrosis, peritonitis, adhesion, bowel obstruction, and sepsis (8). No consensus has yet been reached on POT treatment. Some authors believe that surgical treatment should be performed because of complications that may develop, whereas others argue that late complications are not actually as predicted, and that conservative treatment will be successful. However, surgical resection is easier to relieve pain and the recovery is faster. The duration of the hospital stay is shorter, and the likelihood of developing sepsis is lower. In the medical treatment, the risk of development of sepsis, abscess, and intra-abdominal adhesive band is higher than in the surgical treatment (9). In the recent years, laparoscopic approach is considered to be an effective, safe, and minimally invasive technique for the diagnosis and treatment of POT (10).

\section{Conclusion}

Preoperative diagnosis of torsion of the omentum is hard and is a reason for an acute abdomen. The occurrence of omental torsion should also be kept in mind if the appendix does not disclose the pathology in patients who underwent laparotomy because of the preliminary diagnosis of acute appendicitis. For such patients, alternative approaches, such as paramedian or mid-line incision and diagnostic laparoscopy, should be considered. 
Informed Consent: Written informed consent was obtained from the patient who participated in this case.

Peer-review: Externally peer-reviewed.

Author Contributions: Concept - M.T.K.; Design - M.T.K.; Supervision - M.T.K., I.C.; Resources - M.T.K., O.B.; Materials - M.T.K., I.C.; Data Collection and/or Processing - M.T.K., O.B.; Analysis and/or Interpretation - M.T.K., I.C., O.B.; Literature Search - M.T.K.; Writing Manuscript - M.T.K.; Critical Review - M.T.K.

Conflict of Interest: The authors have no conflict of interest to declare.

Financial Disclosure: The authors declared that this study has received no financial support.

\section{References}

1. Cremonini C, Bertolucci A, Tartaglia D, Menonna F, Galatioto C, Chiarugi $M$. Acute abdomen caused by greater omentum torsion: A case report and review of the literature. Ulus Travma Acil Cerrahi Derg. 2016; 22: 391 4.

2. Occhionorelli S, Zese M, Cappellari L, Stano R, Vasquez G. Acute Abdomen due to Primary Omental Torsion and Infarction. Case Rep Surg. 2014; 2014: 208382. [CrossRef]
3. Borgaonkar V, Deshpande S, Rathod M, Khan I. Primary Omental Torsion Is a Diagnostic Challenge in Acute Abdomen-a Case Report and Literature Review. Indian J Surg. 2013; 75: 255-7. [CrossRef]

4. Tsironis A, Zikos N, Bali C, Pappas-Gogos G, Koulas S, Katsamakis N. Acute abdomen due to primary omental torsion: case report. J Emerg Med. 2013; 44: e45-8. [CrossRef]

5. Karayiannakis AJ, Polychronidis A, Chatzigianni E, Simopoulos C. Primary torsion of the greater omentum: report of a case. Surg Today. 2002; 32: 913-5. [CrossRef]

6. Sencan A, Arslan O, Yilmaz O, Ayhan S, Mir E. A rare cause of acute abdominal pain: primary torsion of omentum majus. Turk J Gastroenterol. 2002; 13: 122-4.

7. Naffaa LN, Shabb NS, Haddad MC. CT findings of omental torsion and infarction: case report and review of the literature. Clin Imaging. 2003, 27: 116-8. [CrossRef]

8. Breunung N, Strauss PA. Diagnostic challenge: Primary omental torsion and literature review - a case report. World J Emerg Surg. 2009; 4: 40. [CrossRef]

9. Mendoza Moreno F, Díez Gago Mdel R, Córdova García DM, Pedraza Muñoz A, Díez Alonso M, Noguerales Fraguas F, et al. Primary omental torsion as presentation of acute abdomen. Case report. Rev Esp Enferm Dig. 2016; 108: 105-6.

10. Hussain K, Munir A, Wahla MS, Mian MA, Masood J. Laparoscopic Management of Primary Segmental Omental Infarction Mimicking Acute Appendicitis. J Coll Physicians Surg Pak. 2015; 25(Suppl 2): 89-90. 